\title{
Thermal Convection in Rotating Horizontal Cylinder Subject to Transverse Vibration
}

\author{
Victor Kozlov $^{1}$, Aleksei Vjatkin', Rustam Sabirov ${ }^{2}$ \\ ${ }^{1}$ Perm State Humanitarian-Pedagogical University \\ Laboratory of Vibrational Hydromechanics \\ 614990, Perm, Sibirskaya av., 24, Russia \\ kozlov@pspu.ru; vjatkin_aa@pspu.ru \\ ${ }^{2}$ Perm National Research Polytechnic University \\ Perm, Russia \\ sabirov@pspu.ru
}

\section{Extended Abstract}

The goal of this research is to study experimentally and theoretically the effect of transversal vibrations on the average convection of a non-isothermal fluid in a rotating cavity [1], especially in the domain where the rotational frequency and vibration frequency approach each other. The research involves experimental studies of the convection of a heat-generating fluid in a rotating horizontal cylinder with isothermal boundaries subject to translational vibrations, perpendicular to the rotation axis [2], as well as thermal convection in an annulus with boundaries at different temperatures. In the absence of vibrations, at fast cavity rotation the fluid is in an equilibrium state in the centrifugal force field with the temperature maximum at the cavity axis. The excitation of average convection and increase of heat transport is discovered when the vibration frequency is near to the rotation frequency. At some discrepancy between the frequencies, the resonance excitation of azimuthal two-dimensional inertial oscillations of the non-isothermal fluid takes place. It results in generation of intensive steady fluid motion and significant growth of heat transfer. The last one results in decrease of the temperature at the cavity axis in case of heat generating fluid. When the frequencies coincide, the vibrations break the axial symmetry of the centrifugal force field. The theoretical analysis of this phenomenon is presented. It is demonstrated, that at this the transversal vibrations result in generation of uniform force field, which is stationary in the cavity frame and provides an intensive thermal convection. The dependence of heat transport on the dimensionless parameters of vibrations is determined both in the case of resonance liquid oscillations and in the case of equal frequencies. The vibrational and the centrifugal mechanisms play the key role in the convection and heat transfer. The transverse vibrations are found to be an efficient tool for the control of thermal convection and heat transfer in rotating systems.

\section{Acknowledgements}

The work was done in the frame of the project № 3.9053.2017/BC and partially supported by the Government of Perm Region (Project C-26/174.9)

\section{References}

[1] V. G. Kozlov, "Thermal Vibrational Convection in Rotating Cavities," Fluid Dynamics, vol. 39, no.1, pp. 3-11, 2004.

[2] A. A. Vjatkin, V. G.Kozlov, R. R. Sabirov, "Convection of heat-generating fluid in a rotating cylindrical cavity subject to transverse vibrations," Int. J. of Thermal sciences, vol. 137, pp. 560-570, 2019. 